\title{
The Quality of Immediate Postnatal Care in Health Facilities in Swaziland: Experience of Postnatal Mothers
}

\section{Bongani R Dlamini ${ }^{*}$, Isabella S Ziyane ${ }^{2}$ and Wendy $\mathbf{P}$ Gule $^{3}$}

${ }^{1}$ Sexual Reproductive Health and HIV, United Nations Population Fund-Swaziland Country Office, Mbabane, Swaziland

${ }^{2}$ Midwifery, University of Swaziland, Mbabane, Swaziland

${ }^{3}$ Midwifery, Southern African Nazarene University, Manzini, Swaziland

\begin{abstract}
Background: The purpose of this study was to evaluate care rendered to HIV positive women during the first six weeks of postpartum. Quantitative, descriptive, cross sectional and analytic study was conducted to investigate postnatal care services provided to HIV positive mothers.

Methods: Data collection was done using structured questionnaires. A total of 372 respondents participated in the study. Descriptive data analysis was used; Epi info version 3.5.1 software was used for data entering and analysis.

Results: The results of the study highlighted that the quality of immediate postnatal care (PNC) was com-promised: only $44.9 \%(n=167)$ had their temperature measured, $26.6 \%(n=99)$ had their pulse rate measured, about half $53.2 \%$ $(n=198)$ were physically assessed following childbirth. Whereas, all the health facilities $(100 \%)$ had the basic equipment for the provision of quality immediate PNC.

Conclusion: The study reveals compromised PNC services provided to HIV positive mothers during the immediate postpartum. It had also documented gaps in the delivery of quality PNC to this special group.

Recommendations: The researchers recommend that the Sexual Reproductive Health Programme within the MoH conduct training needs assessment and skills audit in collaboration with partners, midwifery schools and regulatory bodies to provide evidence-based in-service training and/or specialised trainings in PNC to ensure that a competent midwifery workforce is maintained.
\end{abstract}

Keywords: Immediate postnatal care; Mothers; HIV positive; Midwives; Health facilities; Skilled birth attendants

\section{Introduction}

Approximately 1000 women worldwide die each day from pregnancy related complications. In 2008, alone about 358,000 women died during childbirth. The causes of maternal deaths include delay in obtaining quality PNC services. Although African women attend PNC services, the timing and quality of these services is substandard, evidenced by the high (99\%) maternal mortality rates in developing countries [1]. Sub-Saharan Africa is mostly affected by HIV with a prevalence of 10$<20 \%$.Women of childbearing age bear the brunt of HIV as it accounts for $40 \%$ of people living with HIV globally [2]. Maternal deaths due to HIV and AIDS are as much as $9 \%$ [1]

Swaziland is one of the countries that are greatly affected by the deadly Human-Immuno-deficiency virus (HIV), data from the HIV sero-surveillance report reveals that $41.1 \%$ of antenatal attendees are HIV positive. This situation puts 13,563 infants at risk of acquiring HIV either vertically or postnatal in Swaziland. In an effort to curb Mother to Child Transmission (MTCT) of HIV virus, the Ministry of Health in Swaziland issued guidelines for health practitioners and midwives to standardise practice and offer relevant HIV care, treatment and support to mothers and babies during pregnancy, labour and peuperium [3].

Despite the benefits of postnatal care, gaps in effective delivery of postnatal care services to HIV positive mothers were observed by the researchers in selected health care facilities. This observation was further confirmed by study conducted in 2009 which noted that there was total lack of immediate postnatal care in some underdeveloped countries [4]. Reasons cited by Ziyane and Thwala [5] were personal, socio-cultural, psychological and the quality of postnatal services provided to clients. This is evidenced by high maternal mortality rate at 589 per 100,000 live births in Swaziland [6]. This situation prompted the researcher to inquire about the quality of care rendered to HIV positive mothers during the postnatal period.

\section{Methods}

The objectives of the study were to evaluate immediate postnatal care services provided to HIV positive mothers immediately post-delivery or childbirth. A quantitative, descriptive, cross sectional and analytic study was conducted to investigate immediate PNC services rendered to HIV positive mothers. This approach involved the use of questionnaires as data collecting tools. A non-probability purposive sampling approach was used. The researchers purposely selected HIV positive mothers, who were within the postpartum period who were attending their $6^{\text {th }}$ week PNC services. This was done through the midwives working in PNC units. The researchers checked the antenatal cards as well as the child health card, to ascertain the HIV status of the mothers. The identified mothers were selected. They were informed about the study; their consent both verbal and written was sought. The study population was $372 \mathrm{HIV}$ positive mothers attending first child welfare clinic visit at 6 weeks.

*Corresponding author: Bongani R Dlamini, Sexual Reproductive Health and HIV, United Nations Population Fund-Swaziland Country Office, Mbabane, Swaziland Tel: +26876176844; E-mail: bonganidlamini.bd@gmail.com

Received February 14, 2017; Accepted February 28, 2017; Published March 07 2017

Citation: Dlamini BR, Ziyane IS, Gule WP (2017) The Quality of Immediate Postnata Care in Health Facilities in Swaziland: Experience of Postnatal Mothers. J AIDS Clin Res 8: 672. doi: 10.4172/2155-6113.1000672

Copyright: (c) 2017 Dlamini BR, et al. This is an open-access article distributed under the terms of the Creative Commons Attribution License, which permits unrestricted use, distribution, and reproduction in any medium, provided the original author and source are credited. 
The study was conducted in 9 Public Health Units (PHUs) across the country.

All HIV-positive mothers including their infants, who had come for their 6 week post-natal visit at each of the study sites, were included in the research. All mothers who were HIV-negative as well as those who were HIV-positive but not attending their first 6 week postnatal visit were excluded. The researchers checked the antenatal card as well as the child's health card to ascertain the HIV status of the mothers and the HIV exposure status of the infants as well as the postnatal care visit.

\section{Ethics}

Ethical approval was sought from the Ministry of Health in Swaziland. This included the Swaziland Ethics and Research Committee, the Regional Public Health Unit matrons and senior management at the study settings. The University of South Africa approved the study protocol. Lastly, the participants who took part in the study; their consent was sought verbally and written before each interview.

\section{Results}

\section{Socio-demographic characteristics of respondents}

About three hundred and seventy two mother-infant pair was selected to be part of the study. The women's ages ranged from 15 to 35 years and above. Of the 372 HIV positive women, a majority $(65.8 \%)$ were not formally married (some were cohabiting $(29.8 \%)$, single (29.8\%), widowed (4.0\%), separated (1.9\%) and divorced (0.3\%). As much as $51.8 \%$ had not completed high school and $5.9 \%$ had no formal education. About $51.9 \%$ of the respondents were not employed. As much as $50.8 \%$ were living in peri-urban areas, $37.4 \%$ were residing in rural areas and $11.8 \%$ were living in urban areas (Table 1).

\section{Childbirth attendants}

In Swaziland about $88 \%$ of births are delivered at health facilities, and some still deliver at home. The health facility-based deliveries are attended to by either midwives, doctors or nursing assistants [7]. This study identified that of the $372 \mathrm{HIV}$ positive women, $93.8 \%$ delivered at health facilities and only $6.2 \%$ delivered at home. A majority $(81.7 \%)$ of the respondents were assisted by midwives to deliver their babies, only $10.5 \%$ were managed by doctors and a smaller percentage (1.6\%) was assisted by both doctors and midwives. As much as $6.2 \%$ were delivered by traditional birth attendants (Figure 1).

One of the strategies to reduce maternal mortality rate is to render quality immediate PNC [3]. Provision of quality care immediately after childbirth, can help in assessing and managing risks associated with childbirth to both the mother and neonate. In addition, skilled birth attendants should provide counselling on safe feeding practices. This is critical to HIV positive mothers.

\section{Immediate postnatal care services rendered to HIV positive mothers}

The postnatal period is an equally critical time to emphasize healthy behaviours and care; including early and exclusive breastfeeding, early postnatal family planning, repeat CD4 counts testing and antiretroviral (ARV) prophylaxis or treatment for babies. It also offers the opportunity to address components of preventive essential maternal and newborn care to identify any mothers with problems and address them immediately [8].

The immediate postnatal period is a very important time for the mother, baby and family unit. It is during the first hours after childbirth that the foundations for good recovery, health and adaptation of both mother and baby to their new status are established. Unfortunately, care during this vital postnatal period is often not seen as being as important as antenatal and intrapartum care [9]. The postnatal period is a neglected period, despite the fact that the majority of maternal and new-born deaths occur within the first week of the postnatal period. Health care providers across sub-Saharan Africa continue to advise mothers to come back to the facility for a first check-up only after six weeks [10].

This study revealed that sub-standard PNC was rendered to some respondents as, only $79.8 \%$ of the respondents were measured their blood pressure, $53.2 \%$ undergo physical examination, $26.6 \%$ pulse rate count was done and $44.9 \%$ had their temperature measured immediately after childbirth. This is the time where about $60 \%$ of maternal deaths occur. Such gaps should be avoided, especially if the country is to reduce maternal deaths by three quarters. Moreover, a minority (31.2\%) were asked about ARV drugs that they had taken (Figure 2). Postnatal women are to be given vitamin A; the study found that, only $34.1 \%$ were given Vitamin A. This clearly indicated that the quality of immediate PNC was much compromised, while $60 \%$ of maternal deaths occur during the first $48 \mathrm{~h}$ of peuperium [5]. This finding was consistent with that of [10] who reported that women receive a much compromised PNC, in terms of the essential medical PNC interventions in Africa, especially Sub-Saharan Africa.

Quality immediate PNC include provision of relevant health information to the mother, in a way that she would understand the health messages communicated to her [9]. Exclusive breastfeeding is recommended for all infants in the first six months of life irrespective of their HIV exposure status, as such all mothers should be counselled on the benefits of exclusive breastfeeding during the immediate PNC [3,9]. This study identified that only $58.1 \%$ of the HIV positive women were advised by midwives on exclusive breastfeeding. This finding indicates that midwives are not rendering quality infant feeding education to HIV mothers; who may give early supplementation or offer both

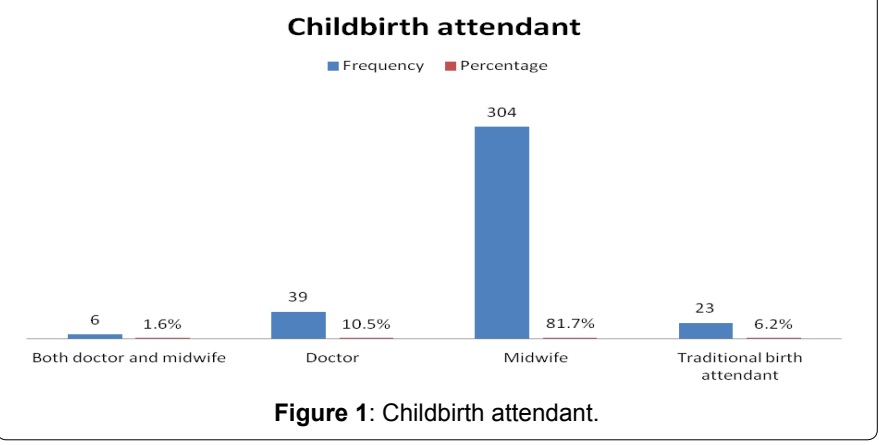

PNC services rendered at discharge

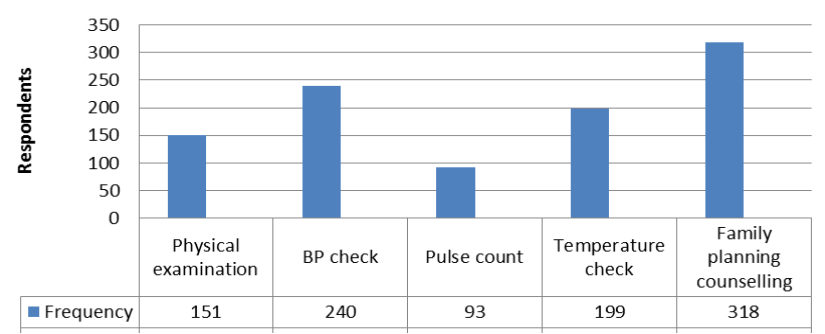

Figure 2: PNC rendered to HIV positive mothers. 


\begin{tabular}{|c|c|c|}
\hline & Frequency & Percent \\
\hline \multicolumn{3}{|l|}{ Age groups } \\
\hline $15-19$ years & 35 & $9.4 \%$ \\
\hline $20-24$ years & 100 & $26.9 \%$ \\
\hline $25-30$ years & 137 & $36.8 \%$ \\
\hline $31-34$ years & 50 & $13.4 \%$ \\
\hline 35 years and above & 50 & $13.4 \%$ \\
\hline \multicolumn{3}{|l|}{ Marital status } \\
\hline Cohabiting & 111 & $29.8 \%$ \\
\hline Divorced & 1 & $0.3 \%$ \\
\hline Married & 127 & $34.1 \%$ \\
\hline Single & 111 & $29.8 \%$ \\
\hline Separated & 7 & $1.9 \%$ \\
\hline Widowed & 15 & $4.0 \%$ \\
\hline \multicolumn{3}{|l|}{ Education level } \\
\hline Tertiary & 26 & $7.0 \%$ \\
\hline Secondary & 135 & $36.3 \%$ \\
\hline Primary & 73 & $19.6 \%$ \\
\hline No formal education & 22 & $5.9 \%$ \\
\hline High school & 116 & $31.2 \%$ \\
\hline \multicolumn{3}{|l|}{ Employment status } \\
\hline Unemployed & 193 & $51.9 \%$ \\
\hline Employed & 179 & $48.1 \%$ \\
\hline \multicolumn{3}{|l|}{ Residential } \\
\hline Urban & 44 & $11.8 \%$ \\
\hline Peri-urban & 189 & $50.8 \%$ \\
\hline Rural & 139 & $37.4 \%$ \\
\hline
\end{tabular}

Table 1: Respondents' socio-demographic information.

breast and replacement feeds with its consequences of mother to child transmission of HIV (MTCT) [3].Women who opt not to breastfeed should be informed of the need to provide nutritionally adequate and safe replacement feeds to enable normal growth and development of the child $[3,11,12]$. Only $39.5 \%$ of the mothers were taught on exclusive replacement feeding. This study reported that $56.2 \%$ of the HIV infected mothers were encouraged to initiate breastfeeding within $1 \mathrm{~h}$. This finding confirms a study that in Swaziland there are areas requiring immediate improvements in terms of PNC, specifically for HIV positive postpartum women [8].

Adherence to ARV medicines cannot be over emphasized. Therefore, every individual using these medicines is to undergo an adherence counselling session not once or twice but every time she comes in contact with midwives [3]. In this study only $38.7 \%$ of HIV positive mothers were counselled on ARV drugs adherence. The individual hygienic conditions during PNC can reduce the vulnerability of complications and infections that can be fatal to either the mother [7]. In this study only $56.2 \%$ were taught on proper hygiene by use of saline sitz baths. This gap in the provision of immediate PNC could result to postpartum infections. This could include ascending infection to the uterus which may result to serious uterine infection [13].

\section{Postnatal care services rendered to HIV positive mothers and their infants at discharge}

Before discharging mothers from health facilities, midwives are to offer counselling. This should include maternal danger signs, infant feeding and personal hygiene. This study reports that only $40.6 \%$ of the HIV-infected mothers were physical examined. About $64.5 \%$ of them had their BP checked, $25.0 \%$ were done pulse rate count assessment, and $53.5 \%$ were checked their temperature before discharge from health facilities (Figure 3). While the MoH states that "no woman should leave

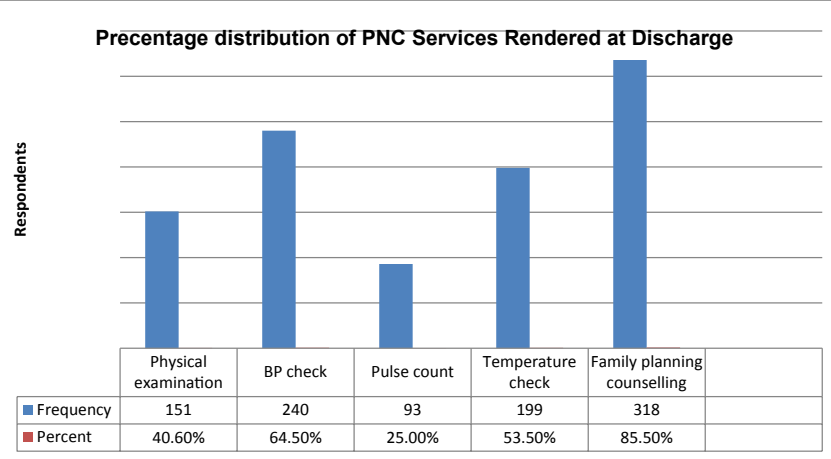

Figure 3: Postnatal care services rendered to respondents at discharge.

maternity without receiving family planning counselling and choosing a method" [3]. A number of HIV positive mothers left health facilities without being assessed.

This study reported that $85.5 \%$ postnatal women were counselled on family planning options. This finding indicates a $14.5 \%$ gap, which indicates provision of poor quality of PNC services rendered to HIV positive mothers, especially because the country is aiming at prevention of unintended pregnancies among HIV positive women [3]. This finding indicates that these women may have unplanned pregnancies, because of midwives' failure to provide family planning counselling.

\section{Health facilities' capacity to render postnatal care services}

Human resources: This study identified that all the facilities (100\%) had midwives working in the PNC units, and all the midwives were oriented to the $\mathrm{MoH} 2010$ guidelines and in all the facilities there were supervisors. Midwives had the knowledge about the recommendations from the $\mathrm{MoH} 2010$ guidelines concerning the key PNC interventions to be rendered to postnatal women [3]. However, the above PNC services that were rendered to HIV infected postnatal mothers did not reveal that these midwives were oriented to the $\mathrm{MoH} 2010$ guidelines. These findings confirms the results of a study conducted in 2008 that shows that generally all health facilities in Swaziland had capacity to offer quality immediate PNC services [8].

Material resource analysis for postnatal care: If quality care is to be rendered the basic material resources should be available, because any shortage is associated with fatal outcomes [14]. Even though almost all the health facilities had the basic material resources, sub-standard care was rendered, indicating midwives' failure to adhere to protocols. This study identified that all the health facilities that were part of this study had all the necessary basic materials to provide quality immediate PNC services. These findings are in line with the Service Availability Mapping Report of the $\mathrm{MoH}$ that reported that basic equipment availability was more than $90 \%$ in all facilities in Swaziland $[6,15]$.

Organizational resources: This study reported that all the health facilities that were involved in this study had antenatal cards, child welfare cards, PNC registers and $\mathrm{MoH} 2010$ National PMTCT guidelines. Generally all facilities in Swaziland have the capacity to provide quality PNC [16]. Facilities have rooms, equipment, medicines and supplies. This study identified that all health facilities that were part of this study had functional maintenance units. This means that any breakage to bio-medical resources could be immediately repaired ensuring that provision of health services could not be interrupted. 


\section{Discussion}

\section{Immediate postnatal care rendered to HIV positive mothers}

Offering quality PNC services can prevent obstetric complications and promote maternal and child health. Antiretroviral therapy can further prolong maternal health and prevent MTCT of HIV among HIV positive women [17]. The seriousness of HIV infection is noted among pregnant women in Swaziland, which currently stand at $41.1 \%$ [3]. Midwives who attend to $57 \%$ deliveries have a responsibility of providing quality immediate PNC interventions [7].

Quality immediate PNC to HIV infected mothers can reduce the risk of complications and deaths as well as promote the health of the mother and the baby [3]. If PNC and curative care in the postnatal period could reach 90 percent of babies and their mothers, about $4 \%$ to $27 \%$ infant mortality could be averted. This implies that quality PNC could save about 310,000 new-born lives a year in Africa [10].

The postnatal period poses substantial health risks for both the mother and the child, more so to HIV infected mothers [1]. Immediate PNC should include complete and systematic physical examination, quality counselling and provision of prophylaxis and family planning [3]. However, the study identified compromised care and gaps in the manner midwives rendered care.

The postnatal period, especially the early phase is also the most neglected part of reproductive health in Africa [10], hence maternal and child mortality is high. Providing quality immediate PNC could improve maternal survival. This would ensure that mothers establish and maintain contact with a number of health services needed during this critical period [18]. Antiretroviral use during pregnancy, labour and postnatal period has proven not only to reduce MTCT of HIV, but also to reduce maternal and infant mortality among HIV positive mothers $[3,19]$.

The immediate postnatal period is a critical period for the foundation of recuperation, adaptation to the environment and for bonding between mother and baby [20]. It is therefore important to render quality care to both mother and baby [9]. Vital signs should be measured to all clients during the critical postnatal care [20]. This includes the temperature, pulse, respirations and the blood pressure. However, the study highlighted compromised PNC and gaps in the manner midwives provided care. Even though vitamin A has been identified as the effective component for health and survival of both the mothers and children, as it can reduce mortality from all causes by approximately 23 percent, as it enhances resistance to disease.Hence it is mandatory for HIV positive post natal clients to receive the treatment. The study identified substandard care offered by midwives.

Exclusive breastfeeding to HIV exposed infants is a recommended feeding practice by $[9,12,13]$. The poor weaning practices which prevail among mothers of infants in Swaziland, forms the basis for the promotion of exclusive breastfeeding [3].This study revealed that some of the HIV positive mothers were not offered quality infant feeding education; who may give early supplementation or offer both breast and replacement feeds with its consequences of MTCT of HIV infection [3], as much as $41.9 \%$ were not educated.

Women should be equipped with knowledge on specific maternal danger signs, so as they could seek medical care as early as possible. This will ensure that complications are identified early before they become life threatening. All women irrespective of HIV status should attend postpartum visit within 3-14 days, so that midwives should assess the maternal readjustment to the pre-pregnant state, and provide PNC services, like ARV prophylaxis, laboratory evaluations (CD4, and haemoglobin level) [21]. This study reported that not all of the women were taught on maternal. This means that women may not visit or go to clinics when experiencing danger signs. This could result to unnecessary maternal deaths.

Generally, post-natal clients are discharged home within the first twenty-four hours post-delivery. Hence, before discharge, mothers should be offered quality PNC and be taught on maternal danger signs: Because of the high risk of death of either the mother or the infant [10].

\section{Postnatal care interventions offered to HIV positive mothers at discharge from health facilities}

Vital signs, especially temperature and pulse blood pressure should be measured and physical examination be performed before discharging post-natal women from health facilities. These would provide valuable clinical information on how the mothers are recuperating from childbirth; ensure identification and management of puerperal problems. Moreover, this will determine the frequency and time mothers should visit PNC clinics [9]. Measuring maternal blood pressure before discharge may help with early identification of signs and symptoms of the second maternal killer cause (pre-eclampsia or eclampsia) among HIV mothers in Swaziland [22]. However, this study identified that only $64.5 \%$ of the respondents had their blood pressure measured, indicating that $34.5 \%$ of HIV-positive mothers did not have their blood pressure measured. This finding revealed a detrimental gap and the provision of compromised PNC by midwives. Failure to measure maternal blood pressure may result in failure to render relevant PNC interventions, for example mothers who had a slight increase in their blood pressure, requiring no treatment but only nutritional counselling on avoidance of foods with high sodium content and the importance of frequent visits to the nearest facilities for close monitoring. Such information may not be provided if the blood pressure is not been measured.

Counting the pulse rate would immediately alert the midwife on puerperal complications which could have an effect on the heart, which might become life threatening within a short space of time. This may also indicate that puerperal infections that may require prophylactic antibiotic treatment were diagnosed [23]. It is expected of midwives to count the pulse rate for mothers before discharging them from their care [3]. However, only $25.0 \%$ of the respondents had their heart rate monitored, meaning that the majority $(75.0 \%)$ were put risk of developing life-threatening puerperal complications, which might result to preventable maternal deaths. This indicates that the quality of obstetric care provided was of poor quality, indicating a life-risk gap in the provision of PNC by midwives.

Measuring HIV-positive mothers' temperature before discharge is critically important in the early identification of puerperal infections. As these women are at risk or they are prone to the development of puerperal and opportunistic infections, because of their low immunity [22]. The temperature result may alert midwives to offer prophylactic antibiotics to mothers with elevated body temperatures, which might indicate puerperal infection. This may also influence the frequency with which such mothers may be expected to visit healthcare facilities, as they require close obstetric monitoring [13]. This study reported that $46.5 \%$ of the HIV-infected mothers who were at higher risk of developing puerperal infections and opportunistic infections were not screened for such possible life-threatening puerperal complications. This indicates another life-threatening gap in the manner midwives provided PNC. This gap may lead to unnecessary HIV infection maternal deaths. 
Physical examination results may help identify mothers who have $3^{\text {rd }}$-degree perineal tears or not contracted uterus that may result in maternal death due to postpartum haemorrhage [20]. HIV-infected women are at risk of having puerperal infection, and might require frequent obstetric care monitoring and provision of prophylactic antibiotics [22]. Therefore, it is of paramount importance that midwives conduct physical examinations, to help avert unnecessary maternal deaths due to preventable causes like postpartum haemorrhage. Surprisingly, only $40.6 \%$ of the HIV positive mothers who were part of the research were physical examined. The majority (59.4\%) were allowed to go home without being offered this crucial PNC intervention. This indicates gross negligence on the part of midwives at the research sites [24], resulting in poor quality of obstetric care received by mothers. The Sexual Reproductive Health Unit (SRHU) should take urgent steps towards addressing this gap in the provision of PNC, especially for HIVpositive mothers because they are prone to puerperal complications as well as opportunistic infections.

Midwives have extensive knowledge on family planning methods. These include barrier, mechanical, chemical and surgical methods of contraception and indications for their use [21]. Moreover, because the Ministry of Health strives to prevent unintended pregnancies among HIV-positive women, midwives should impart their knowledge on family planning options available in the country. They should state that it is available free of charge in public health facilities. This study found that $14.5 \%$ of postnatal mother were not counselled on family planning. This is not acceptable because the country is aiming at the prevention of unintended pregnancies among HIV-positive women [3]. This finding indicates that these women may have unplanned pregnancies, because of midwives' failure to provide family planning counselling.

Before discharge, mothers should be advised and encouraged to attend scheduled PNC visits at healthcare facilities or whenever they notice any danger signs, because of the high risk of death of either the mother or the infant [10]. Women should be equipped with knowledge on specific maternal danger signs, so that they may seek medical care as early as possible. This will ensure that complications are identified early before they become life threatening. It is the task of midwives to provide health education and counselling on maternal danger signs (which include vaginal bleeding, fever, headache and foul-smelling vaginal discharge [21].

This study identified that $28 \%$ of the respondents were not educated on these life-threatening puerperal conditions, which are claiming lives of Swazi women, especially among HIV positive women. This shows poor quality of PNC services rendered and reflects a gap in quality postnatal care provided to HIV positive mothers. This might be contributing to the high maternal mortality rate in Swaziland. The evidence from the study highlighted that generally all the health facilities had the basic material to render quality PNC. This finding is consistent with the findings of several studies $[6,16,25]$. However, substandard care and detrimental gaps were observed.

\section{Limitations of the Study}

While there are many advantages for using questionnaires in research studies, dishonesty can be an issue. Respondents may not be truthful with their answers, due to a number of reasons; such as social desirability bias and attempting to protect gaps in professional practices. As an effort to reduce the risk of dishonesty; the researchers assured respondents that their privacy, confidentiality and anonymity were valued and that the whole data collection, analysis and report writing prevented personal identification. In addition, questionnaires cannot fully capture emotional responses or the feelings of the respondents. The researchers administered the questionnaires face-to-face, to observe and capture facial expression, reactions or body language. Furthermore, open ended questions were included at the end of the questionnaires.

\section{Recommendations and Conclusion}

This study reports compromised PNC services provided to HIV positive mothers during the immediate postpartum. It had also documents gaps in the delivery of quality PNC to this special group. The evidence or findings of this study might inform Maternal New-born Child Health programming in Swaziland. The research recommends a national comprehensive assessment of midwives' skills in providing PNC to HIV-positive mothers and their HIV-exposed infants and a qualitative study to determine the accuracy and the skills of midwives during the provision of PNC services, to help in the development of evidence-based maternal new-born and child health in-service trainings.

\section{References}

1. http://www.who.int/whosis/whostat/2010/en/

2. United Nations Program on HIVIAIDS. Global report: UNAIDS report on the global epidemic. UNAIDS, Geneva 2010.

3. Kingdom of Swaziland Ministry of Health (2010) National prevention of mother to child transmission of HIV guidelines (3rd edn) Mbabane: Printpak 2010.

4. Shongwe LS (2010) The extent of postnatal care delivery at Mbabane Public Health Unit. Bachelor of Nursing Science dissertation. University of Swaziland Mbabane.

5. Ziyane IS, Thwala M (2010) Low uptake of postnatal care in Swaziland. Afr J Midwifery Womens Health 4: 15-21.

6. Kingdom of Swaziland, Ministry of Health (2011) Confidential enquiry into maternal deaths: Triennial report 2008-2010. Mbabane: Webster Print.

7. Kingdom of Swaziland Central Statistical Office (2008) Swaziland 2007 demographic and health survey. Mbabane: Central Statistics Office.

8. Warren C, Shongwe R, Waligo A, Mahdi M, Mazia G, et al. (2008) Repositioning postnatal care in a high HIV environment: Swaziland. Population Council, Washington, DC

9. World Health Organization (2002) Essential antenatal, perinatal and postpartum care: Training modules. WHO, Geneva.

10. Warren C, Daly P, Toure L, Mongi P (2006) Postnatal care. Opportunities for Africa's Newborns.

11. Kramer MS, Kakuma R (2004) The optimal duration of exclusive breastfeeding A systematic review. Adv Exp Med Biol 554: 63-77.

12. World Health Organization (2001) Breastfeeding and replacement feeding practices in the context of mother to child transmission of HIV: An assessment tool for research. WHO, Geneva.

13. Fraser DM and Cooper MA (2009) Myles textbook for midwives. Church Livingstone, New York.

14. Morestin, F, Bicaba, A, Serme, JD, Fournier $P$ (2009) Framework for the quality of obstetric care. BioMed Central.

15. Carla Abou-Zahr (2010) Service availability mapping report. Kingdom of Swaziland Ministry of Health, Webster Print, Mbabane.

16. Mazia G, Narayan I, Warren C, Mahdi M, Chibuye P, et al. (2009) Repositioning postnatal care in a high HIV environment: Swaziland. Glob Public Health 4 253-270.

17. Elizabeth Glaser Paediatric AIDS Foundation (2010) Call to action: End of project report 2002-2009. Elizabeth Glaser Paediatric AIDS Foundation, Washington, DC

18. Sines E, Syed U, Walls S, Worley H (2007) An opportunity to save mothers and children. 
Citation: Dlamini BR, Ziyane IS, Gule WP (2017) The Quality of Immediate Postnatal Care in Health Facilities in Swaziland: Experience of Postnatal Mothers. J AIDS Clin Res 8: 672. doi: 10.4172/2155-6113.1000672

Page 6 of 6

19. World Health Organization (2009) Regional committee for Africa. WHO Geneva.

20. London ML, Ladewig PW, Ball JW, Bindler RC (2007) Maternal and child nursing care (2nd edn) Philadelphia, Pearson Prentice Hall, PA.

21. International Confederation of Midwives (2011) Essential competencies for basic midwifery practice. Hague: ICM.

22. Kingdom of Swaziland Ministry of Health (2011) National comprehensive
HIV package of care: For adults and adolescent in Swaziland. Webster Print Mbabane.

23. Leifer G (2008) Maternity nursing: An introductory text (10th edn) Saunders Elsevier, Philadelphia.

24. United Nations Children's Fund (2007) Vitamin A supplementation: A decade of progress. New York: United Nations Children's Fund.

25. www.healthline.com/galecontent/postpartum 